\title{
Quadratic Program based Control of Fully-Actuated Transfemoral Prosthesis for Flat-Ground and Up-Slope Locomotion
}

\author{
Huihua Zhao ${ }^{1}$ and Aaron D. Ames ${ }^{2}$
}

\begin{abstract}
This paper utilizes a novel optimal control strategy that combines control Lyapunov function (CLF) based model independent quadratic programs with impedance control to achieve flat-ground and up-slope walking on a fully-actuated above-knee prosthesis. CLF based quadratic programs have the ability to optimally track desired trajectories; when combined with impedance control-implemented as a feed-forward term-the end result is a prosthesis controller that utilizes only local information while being robust to disturbances. This control methodology is applied to a bipedal robot with anthropomorphic parameters "wearing" a fully-actuated transfemoral prosthesis. Traditional human-inspired control methods are applied to the human component of the model-simulating nominal human walking-while the novel control method is applied to the transfemoral prosthesis. Through simulation, walking on flat-ground and up-slope is demonstrated, with the resulting gait achieved using the novel prosthesis control yielding walking that is nearly identical to the "healthy" human model. Robustness tests indicate that the prosthesis controller can endure large uncertainties and unknown disturbances.
\end{abstract}

\section{INTRODUCTION}

Impedance control has been a popular approach for the control of both prosthesis [22], [23] and orthosis [15], [13] in recent decades. An ankle orthosis in [12] has been designed using PD control in the swing phase and variable impedance control in the stance phase. Impedance control for a fully-actuated knee-ankle prosthesis can be found in [22]. Combined with using EMG signals for motion intent recognition, impedance control has been successfully applied to an ankle-foot prosthesis in [11]. However, impedance control has problems with maintaining good tracking and robustness, which will be addressed in this work.

While implementing controllers for an amputee, there are basic biomechanical requirements that must be satisfied for a transtibial or transfemoral prosthesis [20], [24]: (1) the prosthetic device must support the body weight of the amputee during the stance phase, i.e., the prosthesis control should provide "stability" during the weight bearing phase; (2) the physical interface between the able body and the prosthesis must prevent undesirable pressure during the locomotion; (3) the prosthesis has to duplicate as nearly as possible the kinematics of a normal gait, i.e., the amputee should walk with normal appearance.

\footnotetext{
*This research is supported by CPS: 1239085, SRI: W31P4Q-13-C-009, CNS: 0953823.

${ }^{1}$ HH.Zhao is with the department of Mechanical Engineering, TAMU, 3128 TAMU, College Station, USA huihuazhaoet amu. edu

${ }^{2}$ Prof. A.Ames is with department of Mechanical Engineering and Electrical Engineering, Texas A \& M University, 3128 TAMU, College Station, USA aames@tamu. edu
}

The goal of this paper is to address requirements (1) and (3) indicated above on a fully-actuated above-knee prosthesis for different types of locomotion. This goal will be achieved via a three steps process. Firstly, traditional human-inspired control methods [5] are reviewed and applied to the human component of the model to obtain human-like locomotion for both flat-ground walking and up-slope walking, based on which, the impedance parameters can be estimated. Then, impedance control is implemented on the prosthetic joints, showing that the estimated parameters are accurate and robust enough to work on the prosthesis. Finally, a novel prosthesis control method: control Lyapunov function based quadratic programs (QPs) coupled with impedance control will be introduced and applied to the prosthesis. The end results indicate improved tracking performance and robustness to unknown disturbances.

Based loosely on the definition of impedance control first proposed by Hogan [14], the torque at each joint during a single step cycle can be represented by a series of passive impedance functions [22]. By reproducing this torque at the prosthetic device joint using the passive impedance functions, one can obtain similar prosthetic gaits compared to those found in normal gait tests. Normally, hand tuning from an expert is required to obtain the impedance parameters [10], [18]; therefore, this leads to another disadvantageit is not optimal. By only using impedance control, we are able to achieve stable robot walking on the prosthetic device (was numerically verified with using Poincarè map). However, considering the drawbacks of impedance control, the obtained tracking performance is lacking, resulting in walking that is not as robust as desired.

Utilizing the impedance controller as a feed-forward term, we present a novel control method that will be utilized as a feedback term to increase robustness and stability. In particular, we begin by considering rapidly exponentially stabilizing control Lyapunov functions (RES-CLFs) as introduced in [8]. This class of CLFs can naturally be stated as inequality constraints in torque such that, when satisfied, rapidly exponential convergence of the error is formally guaranteed. Furthermore, these inequality constraints can be solved in an optimal fashion through the use of quadratic programs. Finally, due to the special structure of the RESCLFs that will be considered in this paper, the CLF based QP can be stated in a model independent fashion. The end result is a novel feedback control methodology: Model Independent Quadratic Programs (MIQP) based upon RESCLFs. These are combined with impedance control to obtain the final prosthetic controller. With the proposed controller, 

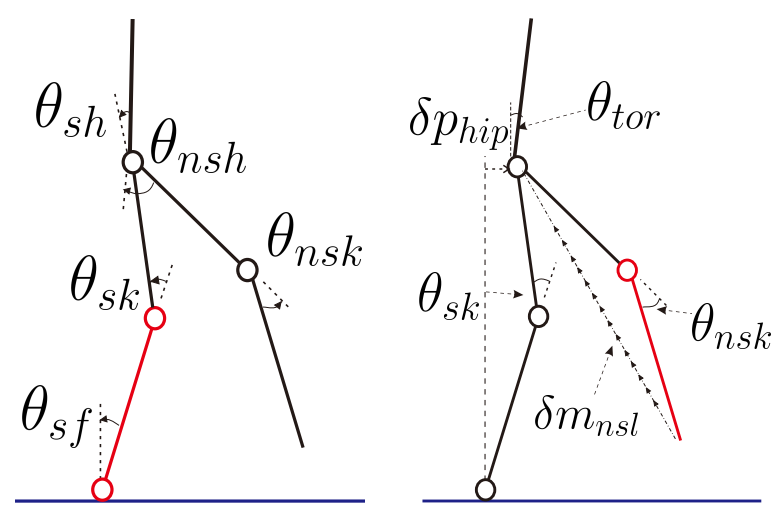

Fig. 1: The angle conventions with prosthetic leg as stance leg (left) and the outputs description with prosthetic leg as swing leg (right).

we will show that the tracking performance can be improved in simulation for both types of locomotion: flat-ground and up-slope walking. In addition, utilizing this novel control method, the robot displays improved stability and robustness to unknown disturbances.

This paper begins with an introduction of the mathematical model of a hybrid system in Sec. II; a brief review of humaninspired optimization is presented for obtaining humanlike walking gaits. Impedance control and the estimation algorithm are discussed in Sec. III. Then the CLF based model independent control will be discussed in detail in Sec. IV. Simulation results using this controller are discussed and compared with using only an impedance controller. Finally, the conclusion will be discussed in Sec. V.

\section{HUMAN-INSPIRED OPTIMIZATION}

In this section, the mathematical model of a bipedal robot is discussed, and the human-inspired control method is introduced so it can be applied to the "human" component of the model. Finally, human-inspired optimization is discussed to obtain human-like flat-ground and up-slope locomotion.

\section{A. Robot Model}

A planar bipedal robot that has five serial chain links (one torso, two thighs and two calves) with anthropomorphic parameters, will be considered as the "human" model in this work. As bipedal robotic walking displays both continuous and discrete behavior (when foot impacts the ground), we formally represent the bipedal robot as a hybrid system [5].

Continuous Dynamics. The configuration space of the robot $Q_{R}$ is described in body coordinates: $\theta=\left(\theta_{s f}, \theta_{s k}, \theta_{s h}, \theta_{n s h}\right.$, $\left.\theta_{n s k}\right)^{T}$ as shown in Fig. 1. The mass and length properties corresponding to the average human model are utilized to derive the equations of motion of the robot, which are given using the Euler-Lagrange formula:

$$
D(\theta) \ddot{\theta}+H(\theta, \dot{\theta})=B u,
$$

where $D(\theta) \in \mathbb{R}^{5 \times 5}$ is the inertial matrix and $H(\theta, \dot{\theta}) \in$ $\mathbb{R}^{5 \times 1}$ contains the terms resulting from the Coriolis effect $C(\theta, \dot{\theta}) \dot{\theta}$ and the gravity vector $G(\theta)$. The torque map $B=I_{5}$ (considering the robot is fully-actuated) and the control, $u$, is the vector of torque inputs. Manipulation of (1) yields the affine control system $(f, g)$ for the continuous dynamics [5].

Discrete Dynamics. When the swing foot hits the ground, a discrete impact will occur, which leads to the velocity changes of the robot, combining with a leg switch simultaneously. With the perfectly plastic impact assumption, the method of [16] is used to obtain the reset map $\Delta_{R}$ as:

$$
\Delta_{R}(\theta, \dot{\theta})=\left[\begin{array}{c}
\Delta_{\theta} \theta \\
\Delta_{\dot{\theta}}(\theta) \dot{\theta}
\end{array}\right],
$$

where $\Delta_{\theta}$ is the relabeling matrix which switches the stance and non-stance leg at impact, and $\Delta_{\dot{\theta}}$ determines the change in velocities due to the impact.

\section{B. Human-Inspired Optimization}

Human-Inspired Outputs. With the goal to achieve humanlike bipedal robotic walking, a human-inspired controller aims to drive the actual robot outputs $y_{a}(\theta)$ to the desired human outputs $y_{d}(t, \alpha)$ which can be represented by the canonical walking function with parameter $\alpha$ [5]. In particular, the outputs considered here are linearized hip position $\delta p_{\text {hip }}$, linearized non-stance slope $\delta m_{n s l}$, two knee angles $\theta_{s k}, \theta_{n s k}$ and the torso angle $\theta_{\text {tor }}$ (see Fig. 1). Therefore, we introduce the human-inspired outputs:

$$
y(\theta, \dot{\theta})=\left[\begin{array}{c}
y_{1}(\theta, \dot{\theta}) \\
y_{2}(\boldsymbol{\theta})
\end{array}\right]=\left[\begin{array}{c}
y_{1}^{a}(\boldsymbol{\theta}, \dot{\theta})-v_{h i p} \\
y_{2}^{a}(\boldsymbol{\theta})-y_{2}^{d}(\rho(\theta), \alpha)
\end{array}\right],
$$

where $y_{1}(\theta, \dot{\theta})$ is the relative degree one output, which is the difference between the actual hip velocity $y_{1}^{a}(\boldsymbol{\theta}, \dot{\theta})$ and the desired hip velocity $v_{\text {hip }} \cdot y_{2}(\theta)$ are the relative degree two human-inspired outputs which are the differences between the actual outputs $y_{2}^{a}(\theta)$ and desired outputs $y_{2}^{d}(\rho(\theta), \alpha)$. Note that, $\rho(\theta)$ is the parameterized time aiming to remove the dependency of time.

With this construction in hand, we represent the dynamics (1) in outputs space as:

$$
\left[\begin{array}{l}
\dot{y}_{1} \\
\ddot{y}_{2}
\end{array}\right]=\underbrace{\left[\begin{array}{c}
L_{f} y_{1}(\theta, \dot{\theta}) \\
L_{f}^{2} y_{2}(\theta, \dot{\theta})
\end{array}\right]}_{L_{f}}+\underbrace{\left[\begin{array}{c}
L_{g} y_{1}(\theta, \dot{\theta}) \\
L_{g} L_{f} y_{2}(\theta, \dot{\theta})
\end{array}\right]}_{A} u,
$$

where $L_{f}$ is the Lie derivative and $A$ is the dynamic decoupling matrix. By picking:

$$
u=A^{-1}\left(L_{f}+\mu\right)
$$

with $\mu$ properly designed as:

$$
\mu=\left[\begin{array}{c}
L_{f} y_{1}(\theta, \dot{\theta}) \\
2 \xi L_{f} y_{2}(\theta, \dot{\theta})
\end{array}\right]+\left[\begin{array}{c}
2 \xi_{y_{1}}(\theta, \dot{\theta}) \\
\xi^{2} y_{2}(\theta)
\end{array}\right],
$$

one can drive both $y_{1} \rightarrow 0$ and $y_{2} \rightarrow 0$ in a provably exponentially stable fashion, which we term the human-inspired controller. Here $\xi$ is the controller gain that determines the convergence rate, details of which can be found in [5].

Partial Hybrid Zero Dynamics. In particular, we are focusing on relative degree two outputs, $y_{2}$, while relaxing relative degree one output to compensate for the model 


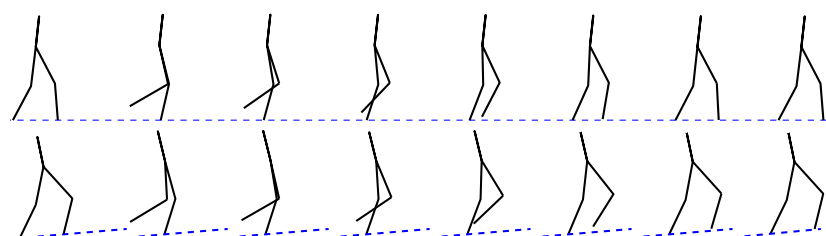

Fig. 2: Gait tiles using human-inspired controller for flatground walking and up-slope walking.

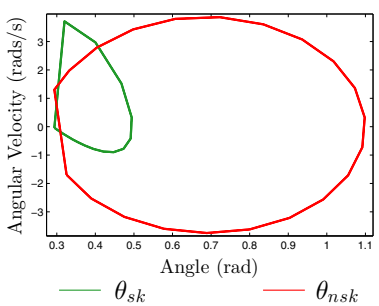

(a) Flat-ground walking

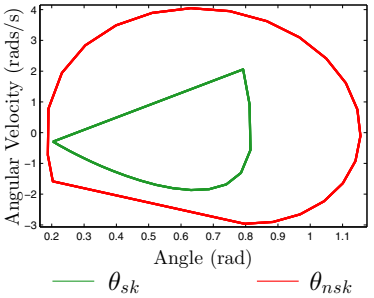

(b) Up-slope walking
Fig. 3: Phase portraits using human-inspired control.

differences between the robot and human. The surface for which these outputs agree all the time is given by the partial zero dynamics surface:

$$
\mathbf{P Z}_{\alpha}=\left\{(q, \dot{q}) \in T Q_{R}: y_{2}(q)=\mathbf{0}, L_{f} y_{2}(q, \dot{q})=\mathbf{0}\right\} .
$$

While the proposed controller (5) renders exponential convergence to this surface, the robot will be "thrown-off" the surface when impacts occur. The goal of partial hybrid zero dynamics (PHZD) is to find parameters $\alpha$ that ensure that this surface remains invariant through impacts: $\Delta(S \cap$ $\mathbf{P Z}(\alpha) \subset \mathbf{P Z}$. This constraint motivates the introduction of an optimization problem that guarantees this condition.

Human-Inspired Optimization. Aiming to find the parameters $\alpha$ yields provably stable human-like robotic walking, an optimization problem subject to PHZD constraints is given:

$$
\begin{aligned}
& \alpha^{*}=\underset{\alpha \in \mathbb{R}^{21}}{\operatorname{argmin}} \operatorname{Cost}_{\mathrm{HD}}(\alpha) \\
& \text { s.t } \quad \Delta\left(S \cap \mathbf{P Z} \mathbf{Z}_{\alpha}\right) \subset \mathbf{P Z}_{\alpha}
\end{aligned}
$$

where the cost function (HIO) is the least-square-fit errors between human experimental data and the CWF representations [5]. Solving the optimization problem discussed above, we can obtain the parameters for both flat-ground walking $\alpha_{l}$ and up-slope walking $\alpha_{r}$, which we term motion primitives (see [26] for more discussions). Importantly, these gaits are provably exponentially stable [6]. The gait tiles for both motion primitives using the human-inspired controller are shown in Fig. 2 and the limit cycles can be seen in Fig. 3. In particular, these results are going to be used as the nominal references of "unimpaired" human walking.

\section{IMPEDANCE CONTROL CONSTRUCTION}

Impedance control will be reviewed at the beginning of this section. Utilizing these concepts, impedance parameter estimation algorithm will be discussed to obtain the impedance parameters automatically for different motion

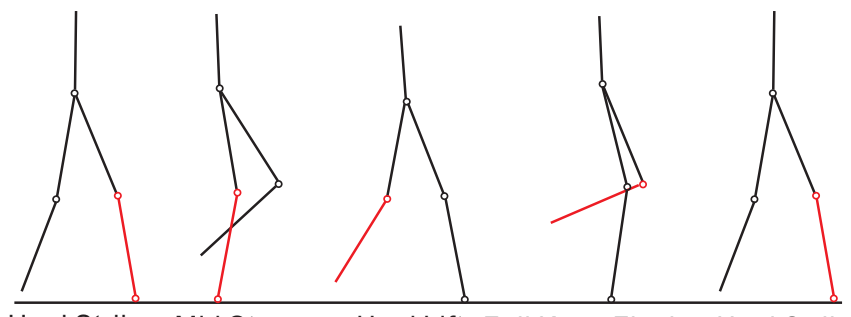

Heel Strike Mid Stance Heel Lift Full Knee Flexion Heel Strike

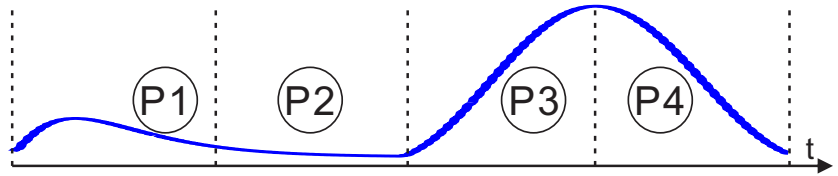

Fig. 4: AMBER gait phase separation. The red line represents the prosthetic device and the red circles are the actuated prosthesis joints. The black lines denote the able body and black cirlces indicate the actuated healthy joints. The green line is the knee angle of one full gait cycle.

primitives. Finally, simulation results of using impedance control will be presented.

\section{A. Impedance Control for Prosthesis}

Impedance Control. Based on the notion of impedance control first proposed by Hogan [14], the torque at each joint during a single step can be piecewisely represented by a series of passive impedance functions with the form:

$$
\tau=k\left(\theta-q^{e}\right)+b \dot{\theta},
$$

where impedance parameters $k, q^{e}$ and $b$ represent the stiffness, equilibrium point and damping respectively, and are constant during specific sub-phases of a particular motion primitive. This formula only requires local informationin this case, the prosthesis knee angle and foot angletherefore, the end result is a simple prosthesis controller [17].

Phase Separation. Based on previous work for flat ground walking [4], analysis of data obtained from the human model shows that one gait cycle can be divided into four phases based on the profile of prosthesis joint angles, which are denoted as $p=1,2,3,4$, and shown in Fig. 4. Specifically, each phase begins at time $t_{0}^{p}$ and ends at $t_{f}^{p}$. The phase separation principle is similar to that in [4] but with different values specific to different motion primitives, which can be referred to in Table I. The impedance torque at the prosthesis joints during a sub-phase $p \in\{1,2,3,4\}$, can be represented by the following equation:

$$
\tau_{p}^{i m p}=k_{p}\left(\theta_{p s}(t)-q_{p}^{e}\right)+b_{p} \dot{\theta}_{p s}(t),
$$

where $\theta_{p s}(t)$ and $\dot{\theta}_{p s}(t)$ denote angles and angular velocities of the prosthesis joints at time $t$. In particular, we have $\theta_{p s}=$ $\left\{\theta_{s f}, \theta_{s k}\right\}$ when the prosthesis leg is the stance leg; and $\theta_{p s}=$ $\theta_{n s k}$ when the prosthesis leg is in the swing phase. Therefore, in each sub-phase $p$, the dynamics of the biped system are governed by the following Euler-Lagrange equations:

$$
\left\{\begin{array}{l}
D(\theta) \ddot{\theta}+H(\theta, \dot{\theta})=B u \quad \forall t \in\left[t_{0}^{p}, t_{f}^{p}\right], \\
\left(\theta\left(t_{0}^{p}\right), \dot{\theta}\left(t_{0}^{p}\right)\right)=R\left(\theta\left(t_{f}^{p-1}\right), \dot{\theta}\left(t_{f}^{p-1}\right)\right),
\end{array}\right.
$$


TABLE I: Estimated Parameters of the Prosthesis Knee Joint.

\begin{tabular}{|c|c|c|c|c|c|c|}
\hline Motion Type & Joints & Phase & Separation & $k_{p}[\mathrm{Nm}]$ & $b_{p}[\mathrm{Nm} s]$ & $q_{b}^{e}[\mathrm{rad}]$ \\
\hline \multirow{6}{*}{ Flat-ground } & \multirow{4}{*}{ Knee } & P1 & heel strike & -574.97 & -133.54 & 0.9867 \\
\hline & & $\mathbf{P 2}$ & $\theta_{s f}<-0.01$ & -642.23 & 8.6826 & 0.3297 \\
\hline & & P3 & heel lift & -5.7679 & 0.3378 & 1.0042 \\
\hline & & P4 & $\dot{\theta}_{n s k}<0$ & -16.186 & 0.5472 & 1.1402 \\
\hline & \multirow{2}{*}{ Ankle } & P1 & heel strike & -574.97 & -133.54 & 0.9867 \\
\hline & & P2 & $\theta_{s f}<-0.01$ & -642.23 & 8.6826 & 0.3297 \\
\hline \multirow{6}{*}{ Up-slope } & \multirow{4}{*}{ Knee } & P1 & heel strike & -338.44 & -4.4349 & 0.2878 \\
\hline & & P2 & $\theta_{s f}<-0.25$ & -216.38 & 17.859 & 0.2024 \\
\hline & & P3 & heel lift & -6 & -0.9626 & 1.1342 \\
\hline & & P4 & $\dot{\theta}_{n s k}<0$ & -25.944 & -0.0634 & 1.2473 \\
\hline & \multirow{2}{*}{ Ankle } & P1 & heel s & -338.44 & -4.4349 & 0.2878 \\
\hline & & P2 & $\theta_{s f}<-0.25$ & -216.38 & 17.859 & 0.2024 \\
\hline
\end{tabular}

where $p$ is set to 4 if $p=1$ and the switching function $\mathrm{R}$ has been defined as the following:

$$
R(\theta(t), \dot{\theta}(t))= \begin{cases}\Delta(\theta(t), \dot{\theta}(t)) & \text { at impacts, } \\ (\theta(t), \dot{\theta}(t)) & \text { otherwise. }\end{cases}
$$

In order to simulate the use of the prosthetic device, for the prosthetic joint, we will replace the corresponding $u_{i}$ term with the prosthetic control input $u_{p s}$ which is $\tau_{p}^{i m p}$ since only the impedance controller is considered, therefore yields the following set up:

$$
D_{i}(\theta) \ddot{\theta}+H_{i}(\theta, \dot{\theta})=\tau_{p}^{i m p} \quad \forall t \in\left[t_{0}^{p}, t_{f}^{p}\right],
$$

where $i$ indicates the $i^{\text {th }}$ row of the corresponding term in (10), which will be updated according to the sub-phase $p$. Note that, we have $i=1,2$ when $p=1,2$, i.e., the prosthetic device is the stance leg, and $i=5$ while $p=3,4$, i.e., the prosthetic device is the non-stance leg.

Impedance Parameter Estimation. In previous work [4], the authors showed that the impedance parameters of the knee joint for a lower-limb prosthesis can be learned by the observation of unimpaired human walking data, which is realized by the human-inspired controller in simulation. The results have been validated both in simulation and in experiment with a transfemoral prosthetic device (video with more details can be seen in [2]). To extend these results, we utilize a similar method to estimate the impedance parameters of multiple joints by observing the simulated "unimpaired" motion primitive data.

We first obtain the "unimpaired" walking data (which are the joint angles and velocities $x=\left(\theta_{p s}, \dot{\theta}_{p s}\right)$ and joint torques $\tau_{p s}$ ) by using the human-inspired controller. By defining the impedance parameter set as $\beta_{p}=\left\{k_{p}, b_{p}, q_{p}^{e}\right\}$ for specific sub-phase $p$, we can form the least square errors minimization problem as follows:

$$
\beta_{p}^{*}=\underset{\beta_{p}}{\operatorname{argmin}} \int_{t_{0}^{p}}^{t_{f}^{p}}\left(\tau_{p}^{i m p}-\tau_{p s, p}\right)^{2} d t,
$$

where $\tau_{p}^{i m p}$ is defined as (9) and $\tau_{p s, p}$ is the "healthy" input torque on the prosthesis joints at sub-phase $p$. By solving this minimization problem, the estimated impedance parameters for each sub-phase can be obtained in Table I.

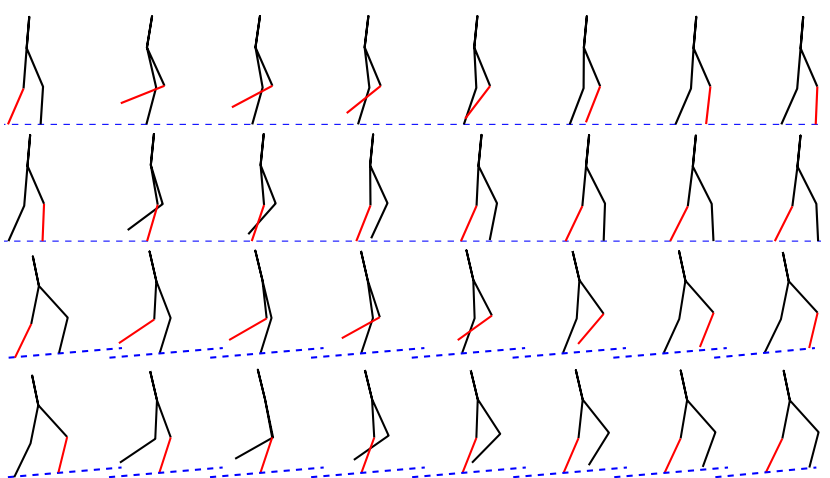

Fig. 5: Gait tiles using impedance control for level walking and slope walking. Red line indicates the prosthesis.
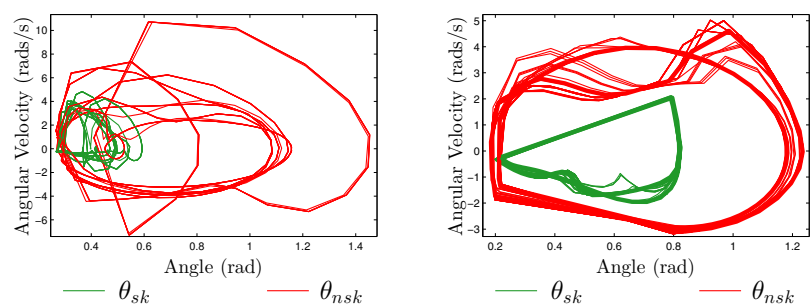

(a) Flat-ground walking phase portrait (b) Up-slope walking phase portrait
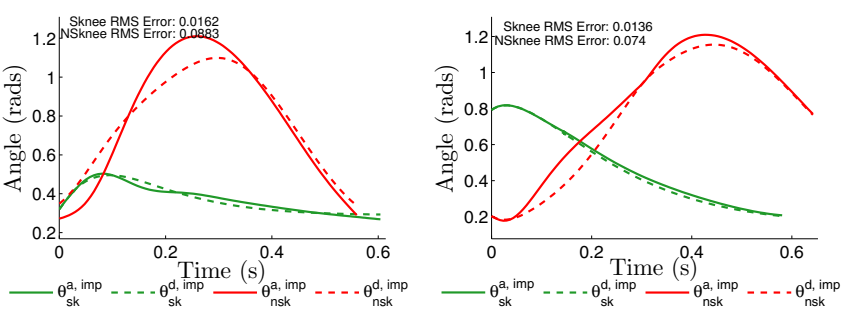

(c) Flat-ground prosthesis knee outputs (d) Up-slope prosthesis knee outputs

Fig. 6: Phase portraits and outputs of the prosthesis knee joint using only impedance control.

\section{B. Simulation Results}

With the obtained impedance parameters, the walking of an amputee "wearing" a tranfemoral prosthesis with an actuated ankle and knee is simulated using only impedance control. The gait tiles in Fig. 5 indicate that good walking has been achieved for both modes of locomotion. The phase portraits for 32 steps of both motion primitives are also shown in Fig. 6a, 6b. The Poincarè map has been utilized to numerically prove that both motion primitives (with prosthesis joints controlled using the impedance controller) are stable with the max eigenvalues less than one (the maximum eigenvalue is 0.34) [19].

\section{MIQP+IMPEDANCE CONTROL}

In this section, we will start with the introduction of rapidly exponentially stabilizing control Lyapunov functions (RES-CLFs) first introduced in [8]. The quadratic programs method is utilized to realize the CLF. When combined with the impedance control (implemented as a feed-forward term), the end result is a novel model independent feedback 
control methodology: Model Independent Quadratic Programs (MIQP)+Impedance control. The simulation results showing better tracking performance, improved stability and robustness, are discussed at the end.

\section{A. CLF MIQP}

As discussed in Sec. II, by designing $\mu$ properly-(6) for example-exponential convergence tracking can be achieved with using the human-inspired controller. This control strategy works well for nonlinear systems if we have good knowledge about the model [21] ; however, this is usually not the case-especially in the case of a prosthesis. PID control still dominates in real world control problems since it does not require accurate model information, i.e., it is model independent. However, considering all the well-known problems of PID control (hand tuning, none optimal [9]), we are motivated to find a new optimal control strategy to overcome these issues while maintaining the model insensitive property.

We begin with substituting the control input (5) to equation (4), which yields the output dynamics in a linear form:

$$
\left[\begin{array}{l}
\dot{y}_{1} \\
\ddot{y}_{2}
\end{array}\right]=\mu \text {. }
$$

By defining the vector $\eta=(y, \dot{y}) \in \mathbb{R}^{n_{1}+2 \times n_{2}}$ with $n_{1}, n_{2}$ denoting the numbers of relative degree one outputs and relative degree two outputs, respectively, equation (14) can be written as a linear affine control system:

$$
\dot{\eta}=\underbrace{\left[\begin{array}{ccc}
0_{n_{1} \times n_{1}} & 0_{n_{1} \times n_{2}} & 0_{n_{1} \times n_{2}} \\
0_{n_{2} \times n_{1}} & 0_{n_{2} \times n_{2}} & I_{n_{2} \times n_{2}} \\
0_{n_{2} \times n_{1}} & 0_{n_{2} \times n_{2}} & 0_{n_{2} \times n_{2}}
\end{array}\right]}_{F} \eta+\underbrace{\left[\begin{array}{cc}
I_{n_{1} \times n_{1}} & 0_{n_{1} \times n_{2}} \\
0_{n_{2} \times n_{1}} & 0_{n_{2} \times n_{2}} \\
0_{n_{2} \times n_{1}} & I_{n_{2} \times n_{2}}
\end{array}\right]}_{G} \mu .
$$

In the context of this control system, we consider the Continuous Algebraic Riccati Equations with $P=P^{T}>0$ :

$$
F^{T} P+P F-P G G^{T} P+I=0,
$$

that yields the optimal solution $\mu=-G^{T} P \eta$. With the aim of achieving stronger bounds of convergence for the considered hybrid system with impacts, we take this method further by defining $\eta_{\varepsilon}=(y / \varepsilon, \dot{y})$ in order to obtain an exponentially stable orbit. Then, we can choose $P$ and $\varepsilon>0$ carefully to construct a rapidly exponentially stabilizing control Lyapunov function (RES-CLF) that can be used to stabilize the output dynamics in a rapidly exponentially fashion [8]. In particular, we define the positive definite CLF as:

$$
V_{\varepsilon}(\eta)=\eta^{T}\left[\begin{array}{cc}
\frac{1}{\varepsilon} I & 0 \\
0 & I
\end{array}\right] P\left[\begin{array}{cc}
\frac{1}{\varepsilon} I & 0 \\
0 & I
\end{array}\right] \eta:=\eta^{T} P_{\varepsilon} \eta
$$

Differentiating this function yields:

$$
\dot{V}_{\varepsilon}(\eta)=L_{F} V_{\varepsilon}(\eta)+L_{G} V_{\varepsilon}(\eta) \mu
$$

where $L_{F} V_{\varepsilon}(\eta)=\eta^{T}\left(F^{T} P_{\varepsilon}+P_{\varepsilon} F\right) \eta, L_{G} V_{\varepsilon}(\eta)=2 \eta^{T} P_{\varepsilon} G$.

In order to exponentially stabilize the system, we want to find $\mu$ such that, for specifically chosen $\gamma>0$ [8], we have:

$$
L_{F} V_{\varepsilon}(\eta)+L_{G} V_{\varepsilon}(\eta) \mu \leq-\frac{\gamma}{\varepsilon} V_{\varepsilon}(\eta)
$$

Therefore, an optimal $\mu$ could be found by solving the following quadratic programs $(\mathrm{QP})$ as:

$$
\begin{aligned}
& m(\eta)=\underset{\mu \in \mathbb{R}^{n_{1}+n_{2}}}{\operatorname{argmin}} \mu^{T} \mu \\
& \quad \text { s.t } \varphi_{0}(\eta)+\varphi_{1}(\eta) \mu \leq 0,
\end{aligned}
$$

where $\varphi_{0}(\eta)=L_{F} V_{\varepsilon}(\eta)+\frac{\gamma}{\varepsilon} V_{\varepsilon}(\eta)$ and $\varphi_{1}(\eta)=L_{G} V_{\varepsilon}(\eta)$.

Note that, instead of substituting the optimal solution $\mu$ into equation (5) to obtain the feedback control law as in [7], we use $\mu$ directly as a control input into the original system without considering the model decoupling matrix $A$ and $L_{f}$. Therefore, we term this strategy Model Independent Quadratic Programs (MIQP) control.

Taking a further look into the MIQP algorithm, we basically constructed a new linear control system (15) that only focuses on the errors between the actual outputs and desired outputs, while not requiring any information about the original model. Another immediate advantage is that torque bounds can be directly applied in this formulation; thus, the optimal control value can be obtained while respecting the torque bounds. As discussed in [7], this can be achieved by relaxing the CLF constraints with a large penalty value $p>0$. In particular, we consider the MIQP as:

$$
\begin{aligned}
& \underset{(\delta, \mu) \in \mathbb{R}^{n_{1}+n_{2}+1}}{\operatorname{argmin}} p \delta^{2}+\mu^{T} \mu \\
& \text { s.t } \quad \varphi_{0}(\eta)+\varphi_{1}(\eta) \mu \leq \delta, \\
& \mu \leq \mu_{M A X}, \\
& -\mu \leq \mu_{M A X} \text {. }
\end{aligned}
$$

By solving this QP problem, an optimal controller can be obtained to regulate the output dynamics with a rapidly exponentially stable fashion for the bipedal robot model.

\section{B. MIQP+Impedance Control}

While MIQP control benefits from its model independent property in an optimal fashion, it also suffers from the overshoot problem as with PID controller because of the lack of model information. Particularly, overshoot issue will be a fatal problem for a prosthesis controller with the safety consideration of an amputee; this motivates the introduction of MIQP+Impedance control.

By considering the impedance control $\tau^{i m p}$ as the feedforward term, the input torque $u_{p s}$ of the prosthetic joints can be stated as the following:

$$
u_{p s}=\tau^{q p}+\tau^{i m p},
$$

where $\tau^{q p}$ is the torque computed from the MIQP problem. Taking the idea further, we add the impedance term $\tau^{i m p}$

TABLE II: RMS Errors with Using Different Controllers.

\begin{tabular}{c|cc|cc}
\hline Motion & \multicolumn{2}{|c}{ Flat-ground } & \multicolumn{2}{c}{ Up-slope } \\
\hline Joints & Impedance & MIQP+Impedance & Impedance & MIQP+Impedance \\
\hline$\theta_{s f}$ & 0.0181 & 0.0053 & 0.0174 & 0.0033 \\
$\theta_{s k}$ & 0.0162 & 0.0017 & 0.0136 & 0.0007 \\
$\theta_{n s k}$ & 0.0883 & 0.0009 & 0.074 & 0.0045 \\
\hline
\end{tabular}




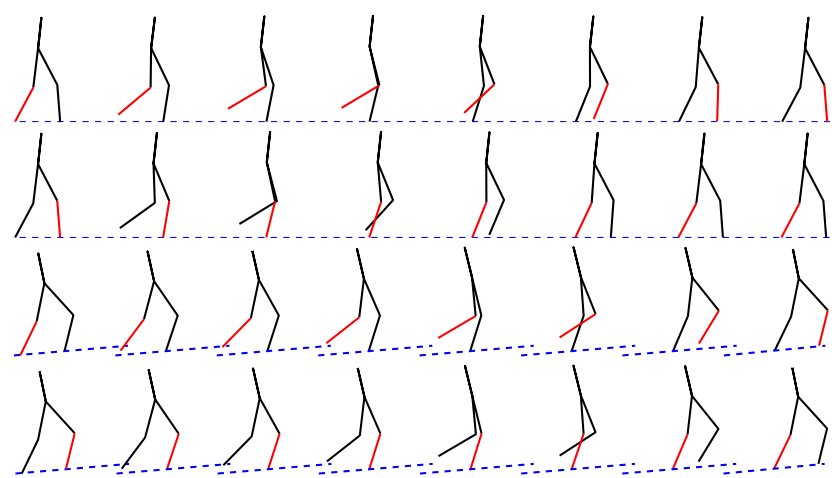

Fig. 7: Gait tiles using MIQP+Impedance control for level walking and slope walking. Red line indicates the prosthesis.

into the MIQP construction, which yields the following MIQP+Impedance problem:

$$
\begin{array}{rlrl}
\underset{\left(\delta, \tau^{q p}\right) \in \mathbb{R}^{n_{1}+n_{2}+1}}{\operatorname{argmin}} p \delta^{2}+\tau^{q p T} \tau^{q p} & \\
\text { s.t } \varphi_{0}(\eta)+\varphi_{1}(\eta) \tau^{q p} \leq \delta-\varphi_{1}(\eta) \tau^{i m p}, \quad \text { (CLF) } \\
\tau^{q p} \leq \tau_{M A X}^{q p}, & \text { (Max QP Torque) } \\
-\tau^{q p} & \leq \tau_{M A X}^{q p}, & & \text { (Min QP Torque) } \\
\tau^{q p} & \leq \tau_{M A X}-\tau^{i m p}, & \text { (Max Input Torque) } \\
-\tau^{q p} & \leq \tau_{M A X}+\tau^{i m p} . & & \text { (Min Input Torque) }
\end{array}
$$

By adding the impedance control as a feed-forward term into the input torque, the model independent dynamic system (15) gathers some information about the system that it is controlling. It can, therefore, adjust $\tau^{q p}$ accordingly to accommodate the feed-forward term in order to achieve exceptional tracking. By setting the QP torque bounds $\tau_{M A X}^{q p}$, we can limit the overshoot problem. Note that, we also set the total input torque bounds for the QP problem such that the final optimal input torque (22) will satisfy the total torque bounds, which is critical for practical implementation.

\section{Simulation Results}

Considering the different degrees of actuation applied in the stance and non-stance phases, the MIQP+Impedance controller has to be constructed accordingly. In particular, during the phase in which the prosthetic device is the stance leg, both knee and ankle are actuated; therefore, the outputs we choose are the linearized hip position $\delta p_{\text {hip }}$ and the stance knee angle $\theta_{s k}$, i.e., we have $n_{1}=1$ and $n_{2}=1$. For the phase in which the prosthetic device is the swing leg, only the prosthesis knee joint is actuated; thus, the output $\theta_{n s k}$ is chosen, i.e., $n_{1}=0$ and $n_{2}=1$.

Tracking Performance. The gait tiles of both flat-ground walking and up-slope walking are shown in Fig. 7. The tracking results of the prosthesis knee joint for both motion primitives are shown in Fig. 8a and 8b, respectively. The tracking $R M S$ errors are all less than $0.005 \mathrm{rad}$, which is at least 3 times better than just using impedance control as shown in Fig. 6a and 6b. Tracking errors of all the prosthesis joints are shown in Table II explicitly. The phase portraits
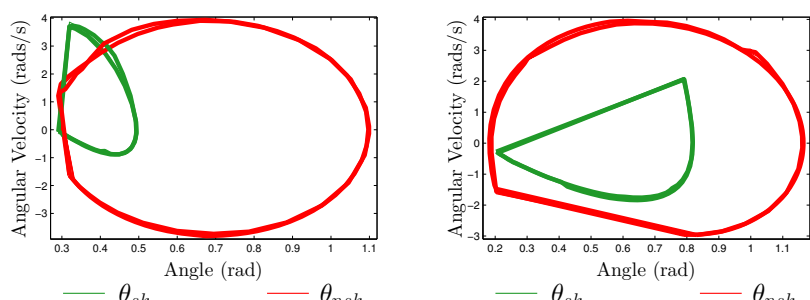

(a) Flat-ground walking phase portrait (b) Up-slope walking phase portrait
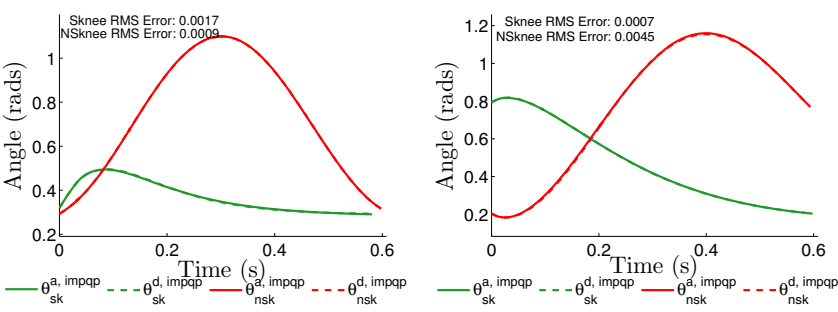

(c) Flat-ground prosthesis knee outputs (d) Up-slope prosthesis knee outputs

Fig. 8: Phase portraits and outputs of the prosthesis knee joint with MIQP+Impedance control.

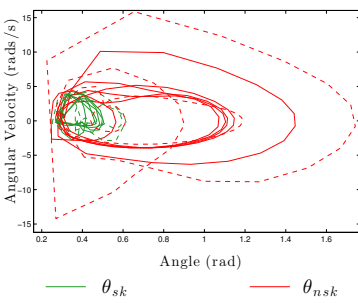

(a) Impedance Control.

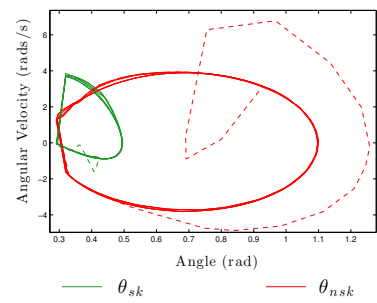

(b) MIQP+Impednce Control.
Fig. 9: Phase portrait of $8 \mathrm{~N}$ impulse disturbance rejection. The dash lines represent the disturbances.

for 32 steps of both the motion primitives can be seen in Fig. 8 . Note that, compared to the phase portraits with using the human-inspired controller in Fig. 3, even though we have asymmetric controllers on the two knee joints, the phase portraits converge to nearly one limit cycle quickly. That is to say, with the proposed controller, the prosthesis can duplicate the kinematics of the normal gait, i.e., the amputee can achieve walking gaits that are nearly identical to unimpaired human walking.

Robustness Tests. Stability is another critical requirement for a lower-limb prosthesis controller. The prosthetic device must be robust and stable enough to endure unknown environment disturbances. In simulation, we show that the proposed MIQP+Impedance controller renders more robustness to the prosthetic device than only using impedance control, and therefore, is safer for the amputees' daily use.

1) Reaction to impulse push: Impulse forces (lasting for $0.01 \mathrm{~s}$ ) have been applied to the prosthetic leg during both stance and non-stance phases. The maximum impulse force that the robot can tolerate and maintain good tracking with the proposed MIQP+Impedance controller is $30 \mathrm{~N}$. However, 
when using only impedance control, the maximum force the robot can endure is $10 \mathrm{~N}$; moreover, the tracking becomes worse after the disturbance and, as a result, leads to a failure to walk eventually.

In order to compare the disturbance rejection properties of the two controllers more explicitly, we apply the same impulse force $(8 \mathrm{~N})$ on the prosthetic knee joint that are controlled with different controllers. The phase portraits for 8 steps of using these two controllers are shown in Fig. 9, from which, we can see that the phase portrait of MIQP+Impedance control converges back to the limit cycle quickly and stably. However, the phase portrait for the controller using only impedance control shows large velocity spikes and can not converge to the original periodic orbit. A video [3] is attached to show the details.

2) To overcome an obstacle: In the simulation, we let the robot walk over a $20 \mathrm{~mm}$ obstacle on flat ground. The gait tiles can be seen in Fig. 10, which shows that the robot can overcome the obstacle smoothly and keep walking normally using the MIQP+Impedance control. A similar test is also conducted with only using the impedance control. The robot can also walk over the obstacle, however, the tracking performance using only the impedance controller is worse, the details of which can be seen in the video [3].

\section{CONCLUSIONS}

Utilizing the human model with anthropomorphic parameters "wearing" a fully-actuated above-knee prosthesis, we successfully implemented a novel optimal control strategy on this testbed to achieve flat-ground and up-slope locomotion. The proposed MIQP+Impedance controller benefits from both the feed-forward impedance control that gives us model information and the MIQP method that renders model independence in an optimal fashion via the CLF based QP control methods. This approach has already been applied to a physical robot AMBER with only the knee joint being actuated. The experimental results showed improved tracking performance, stability, and robustness to unknown disturbances [25] (more details can be seen in video [1]). The limitation of this work is that only a point foot model is considered, which limits the ability to capture the whole picture of human locomotion. A more sophisticated model with heel lift and toe strike will be considered to test the proposed MIQP+Impedance controller in future work.

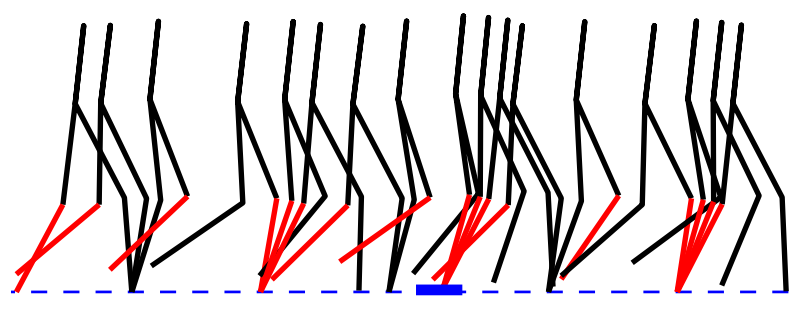

Fig. 10: Gait tiles of walking over a $20 \mathrm{~mm}$ obstacle with MIQP+Impednce Control.

\section{REFERENCES}

[1] CLF based MIQP+Impedance Control on AMBER. http://youtu.be/5TuTyKhMNiU.

[2] Learning Impedance Controller Parameters for Lower-Limb Prostheses. http://youtu.be/AzF5-gqtRbc.

[3] Video of Simulation Results. http: / / youtu.be/Z0oWlYwgD-A.

[4] Navid Aghasadeghi, Huihua Zhao, Levi J Hargrove, Aaron D Ames, Eric J Perreault, and Timothy Bretl. Learning impedance controller parameters for lower-limb prostheses. IEEE: IROS, 2013.

[5] A. D. Ames. First steps toward automatically generating bipedal robotic walking from human data. In 8th International Workshop on Robotic Motion and Control, RoMoCo'11, Bukowy Dworek, 2011.

[6] A. D. Ames. Human-inspired control of bipedal robots. In Conditionally accepted for publication in IEEE Transactions on Automatic Control, 2012.

[7] A. D. Ames. Human-inspired control of bipedal robots via control lyapunov functions and quadratic programs. In Proceedings of the 16th international conference on Hybrid systems: computation and control, pages 31-32. ACM, 2013.

[8] A. D. Ames, K. Galloway, and J.W. Grizzle. Control lyapunov functions and hybrid zero dynamics. In Decision and Control (CDC), 2012 IEEE 51st Annual Conference on, pages 6837-6842, 2012.

[9] D.P. Atherton and S. Majhi. Limitations of pid controllers. In American Control Conference, pages 3843-3847, 1999.

[10] Christopher G Atkeson and Stefan Schaal. Robot learning from demonstration. In ICML, volume 97, pages 12-20, 1997.

[11] Samuel Au, Max Berniker, and Hugh Herr. Powered ankle-foot prosthesis to assist level-ground and stair-descent gaits. Neural Networks, 21(4):654 - 666, 2008.

[12] J.A. Blaya and H. Herr. Adaptive control of a variable-impedance ankle-foot orthosis to assist drop-foot gait. Neural Systems and Rehabilitation Engineering, IEEE Transactions on, 12(1):24-31, 2004.

[13] A.W. Boehler, K.W. Hollander, T.G. Sugar, and Dosun Shin. Design, implementation and test results of a robust control method for a powered ankle foot orthosis (afo). In Robotics and Automation, ICRA, IEEE, pages 2025-2030, 2008.

[14] Neville Hogan. Impedance control: An approach to manipulation. pages 304-313, 1984.

[15] K.W. Hollander and T.G. Sugar. A robust control concept for robotic ankle gait assistance. In Rehabilitation Robotics, ICORR, IEEE 10th International Conference on, pages 119-123, 2007.

[16] Y. Hürmüzlü and D. B. Marghitu. Rigid body collions of planar kinematic chains with multiple contact points. Intl. J. of Robotics Research, 13(1):82-92, February 1994.

[17] René Jimenez-Fabian and Olivier Verlinden. Review of control algorithms for robotic ankle systems in lower-limb orthoses, prostheses, and exoskeletons. Medical engineering \& physics, 34(4):397-408.

[18] Jun Nakanishi, Jun Morimoto, Gen Endo, Gordon Cheng, Stefan Schaal, and Mitsuo Kawato. Learning from demonstration and adaptation of biped locomotion. Robotics and Autonomous Systems, 47(2):79-91, 2004.

[19] T.S. Parker and L.O. Chua. Practical numerical algorithms for chaotic systems. 1989.

[20] Dejan Popovic, Rajko Tomovic, Dejan Tepavac, and Laszlo Schwirtlich. Control aspects of active above-knee prosthesis. International Journal of Man-Machine Studies, 35(6):751 - 767, 1991.

[21] S. S. Sastry. Nonlinear Systems: Analysis, Stability and Control. Springer, New York, June 1999.

[22] Frank Sup, Amit Bohara, and Michael Goldfarb. Design and Control of a Powered Transfemoral Prosthesis. The International journal of robotics research, 27(2):263-273, February 2008.

[23] Toshio Tsuji, Keisuke Shima, Nan Bu, and Osamu Fukuda. Biomimetic impedance control of an emg-based robotic hand.

[24] Radcliffe C. W. Biomechanical basis for the design of prosthetic knee mechanisms. Rehabilitation Engineering International Seminar, 1980.

[25] H. Zhao, S. Kolathaya, and A. D. Ames. Quadratic programming and impedance control for transfemoral prosthesis. In Robotics and Automation (ICRA), IEEE International Conference, 2014.

[26] Huihua Zhao, Mathew Powell, and Aaron D. Ames. Human-inspired motion primitives and transitions for bipedal robotic locomotion in diverse terrain. Optimal Control Applications and Methods, 2013. 